\title{
Approaching the Theoretical Limit of Diamagnetic-Induced Momentum in a Rapidly Diverging Magnetic Nozzle
}

\author{
Kazunori Takahashi, ${ }^{1,2, *}$ Christine Charles, ${ }^{2}$ and Rod W. Boswell ${ }^{2}$ \\ ${ }^{1}$ Department of Electrical Engineering and Computer Science, Iwate University, Morioka 020-8551, Japan \\ ${ }^{2}$ Space Plasma, Power and Propulsion Laboratory, Research School of Physics and Engineering, The Australian National University, \\ Canberra ACT 0200, Australia
}

(Received 15 February 2013; published 8 May 2013)

\begin{abstract}
Cross-field diffusion and plasma expansion in a rapidly diverging magnetic nozzle are controlled while maintaining constant plasma production in a contiguously attached radio frequency plasma source. It is demonstrated that the measured electron-diamagnetic-induced axial momentum increases with increasing magnetic field strength to approach the theoretical limit derived using an ideal nozzle approximation. The measured axial momentum exerted onto the axial and radial plasma source boundaries validate the prediction from a maximum electron pressure model on the back wall and from a zero net axial momentum model on the radial wall.
\end{abstract}

DOI: 10.1103/PhysRevLett.110.195003

PACS numbers: 52.30.Ex, 52.72.+v, 52.75.Di

Momentum transport, ion heating, waves, and turbulence in magnetized collisionless plasmas are recurrent themes for investigation of space structures (i.e., the solar corona [1] and the astrophysical jet [2]) or space events (i.e., magnetic reconnection [3]). Although the multiplicity of time scales in space events such as aurorae, solar flares, and astrophysical jets may not be amenable to steady state solutions, a diverse number of opinions and models that attempt to unify the sparse data can be found [4]. Some theoretical aspects of momentum imparted by a magnetic nozzle and plasma detachment from a magnetic field have been discussed in the context of space physics [5] and of electric propulsion [6-8]. Fruchtman has discussed various effects such as ambipolar and nonambipolar cross-field diffusions for a two-dimensional steady state plasma simply confined by an axial magnetic field [9] and double layers in a plasma flow along a divergent magnetic field [5]. Ahedo and Merino have described momentum in a convergent-divergent nozzle by computing electron and ion flows in two dimensions and the resultant current profile [7]. Arefiev and Breizman have solved the ideal magnetohydrodynamic equations for a cold plasma flow in a slowly diverging nozzle [6]. More recently, Rao and Singh [10] have reported two-dimensional particlein-cell simulations of a steady state, uniform plasma injection into a rapidly divergent magnetic nozzle.

The design of controlled laboratory experiments is complex since important parameters such as magnetic field strength and structure and plasma generation and flow are often nonlinearly coupled, and some of the scaling is very different as is always the case when comparing laboratory and space experiments. The scaling problem also exists when comparing computer simulations $[5,10]$ to space experiments. Most theoretical studies address either plasma momentum in unmagnetized cylindrical bounded plasmas with no radial momentum loss [11] or plasma momentum imparted by a magnetic nozzle with constant and uniform plasma injection $[5,7,10,12]$. Initial experiments have shown the presence of an electron diamagnetic effect on the axial plasma momentum imparted by the magnetic nozzle and of plasma momentum imparted from a geometrically bounded plasma [13]. These provide some insight into the physics of magnetic nozzle plasmas but the theoretical developments have not yet been fully experimentally verified due to the restrictive set of assumptions and the difficulty in obtaining independent control of plasma production and expansion.

Here a laboratory experiment is designed to provide direct measurement of axial plasma momentum in a rapidly diverging magnetic nozzle for a constant low-beta plasma injection into the field structure. The strength of the nozzle field is increased to inhibit cross-field diffusion and to approach the theoretical limit of diamagneticinduced momentum for an ideal magnetic nozzle. In addition, the axial momentum components respectively exerted on the axial and radial boundaries are independently measured. The experiment is subsequently tuned to verify that an asymmetry of the axial density profile to the left or to the right of the plasma cavity center of gravity generates a net axial momentum imparted to the radial boundary of opposite signs, thereby validating the momentum diagnostic and extending the theoretical study.

The previously described pendulum force balance [13] and a presently designed plasma source with a rapidly diverging magnetic nozzle [Fig. 1(a)] are immersed in a 60-cm-diameter and 140-cm-long vacuum chamber, which is evacuated to a base pressure of $10^{-6}$ Torr. The system consists of a $6.4-\mathrm{cm}$-diameter and $20-\mathrm{cm}$-long glass source tube, a solenoid for the magnetic nozzle formation, a radio frequency (rf) antenna, and a source back plate mechanically independent of the cylindrical tube and with a small center hole. The axial position of the source exit is defined as $z=0$. Argon gas of $24 \mathrm{sccm}$ flow rate is introduced into 


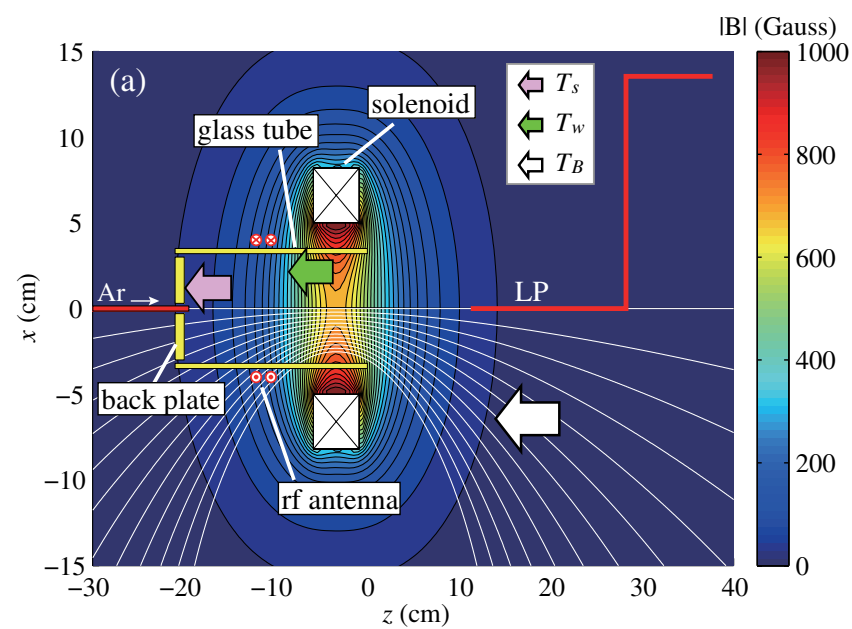

(b)

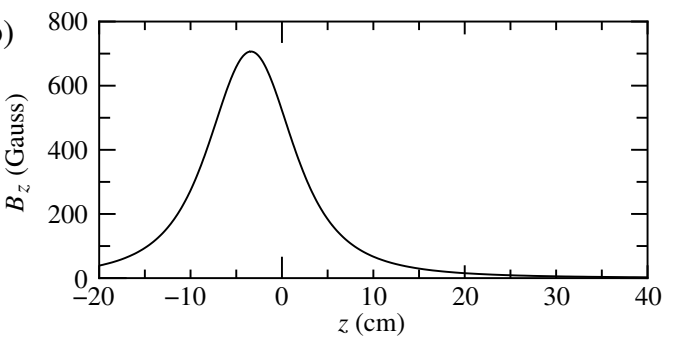

(c)

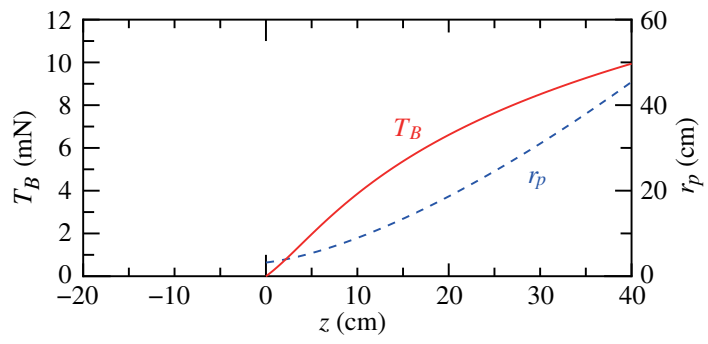

FIG. 1 (color online). (a) Schematic diagram of the plasma source. The contour color shows the calculated magnetic field strength $|B|$ in Gauss for $I_{B}=14 \mathrm{~A}$, and the white lines show the magnetic field lines. (b) Axial profile of the calculated magnetic field $B_{z}$ on axis for $I_{B}=14 \mathrm{~A}$. (c) Axial profiles of the force $T_{B}$ (solid line) and plasma radius $r_{p}$ (dashed line) calculated from the one-dimensional ideal magnetic nozzle approximation.

the source tube through the small center hole of the back plate $\left(z_{\text {end }}=-20 \mathrm{~cm}\right)$, resulting in a chamber pressure of 0.8 mTorr. The double-turn rf loop antenna $(z=$ $-11.5 \mathrm{~cm}$ ), shielded as described in Ref. [14] to inhibit the formation of any parasitic discharge outside the source tube, is powere from a $13.56 \mathrm{MHz}$ and $1 \mathrm{~kW}$ rf generator to produce a stable partially ionized argon plasma. The solenoid current $I_{B}$ can be increased up to $14 \mathrm{~A}$, yielding a maximum magnetic field strength of $710 \mathrm{G}$ at the solenoid central position $\left(z_{\mathrm{sol}}=-3.5 \mathrm{~cm}\right)$ near the source exit and rapidly decreasing to $175 \mathrm{G}$ at the rf antenna position $(z \sim-11.5 \mathrm{~cm})$; the calculated $x-z$ and $z$ profiles of the magnetic field strength for $I_{B}=14 \mathrm{~A}$ are shown by color contours in Figs. 1(a) and 1(b), respectively, together with the field lines (white lines) in Fig. 1(a).
The plasma density is measured using a 3-mm-diameter planer Langmuir probe [LP in Fig. 1(a)], assuming a Maxwellian electron energy distribution and taking into account the sheath expansion effect [15]. The probe has a dog-leg shaft mounted on a computer-controlled steppingmotor system immersed in vacuum, which allows an accurate control of the probe positions. Figure 2(a) shows the axial profiles of the plasma density on axis as a function of $I_{B}$. For all values of $I_{B}$, the profiles have a maximum density near the $\mathrm{rf}$ antenna position of $z \sim-12 \mathrm{~cm}$. The density on axis for no magnetic field $\left(I_{B}=0 \mathrm{~A}\right)$ is asymmetric between the regions from the maximum density position to left and to right, while it becomes more symmetric along $z$ within the source cavity when applying the magnetic field. Above $2 \mathrm{~A}$, no measurable change of density in the source tube is seen on Fig. 2(a). The measured electron temperature (not shown here) is uniform on axis within the source cavity $(z<0)$ at about $6-7 \mathrm{eV}$, but gradually decreases along the $z$ axis to values of $3-4 \mathrm{eV}$ at $z \sim 30 \mathrm{~cm}$, and this decrease is invariant with the magnetic field strength. Figure 2(b) shows the $x$ profile of the plasma density measured at the axial position of maximum density $(z=-12 \mathrm{~cm})$ for $I_{B}=5 \mathrm{~A}$ (filled squares) and $14 \mathrm{~A}$ (open circles) with no noticeable change between the two
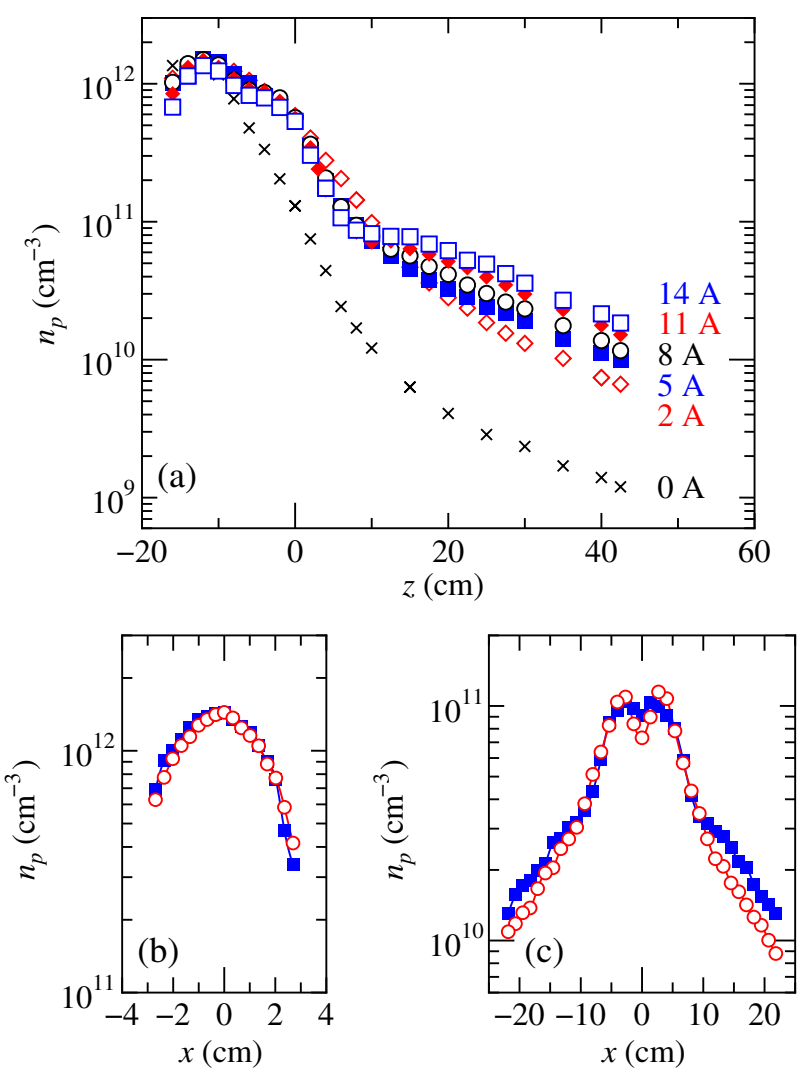

FIG. 2 (color online). (a) Axial profiles of the plasma density on axis with $I_{B}$ as a parameter. $x$ profiles of the (b) upstream $(z=$ $-12 \mathrm{~cm})$ and $(\mathrm{c})$ downstream $(z=8 \mathrm{~cm})$ plasma densities for $I_{B}=5 \mathrm{~A}$ (filled squares) and $14 \mathrm{~A}$ (open circles). 
cases. The ion Larmour radius at the rf antenna position $(z=-11.5 \mathrm{~cm})$ decreases from $\sim 7 \mathrm{~cm}$ at $I_{B}=5$ A to $\sim 2.5 \mathrm{~cm}$ at $I_{B}=14 \mathrm{~A}$, which is larger than or comparable to the source radius $(3.2 \mathrm{~cm})$, where a singly charged argon ion with a temperature of $0.2 \mathrm{eV}$ is assumed for the calculation. The results on Figs. 2(a) and 2(b) show that plasma production is unchanged with increasing solenoid current since the upstream field is maintained below $175 \mathrm{G}$ and does not significantly affect the plasma loss rate onto the source tube radial wall. The density in the magnetic nozzle downstream of the source is found to increase with an increase in $I_{B}$ as seen in Fig. 2(a) as a result of inhibition of the cross-field diffusion by the stronger magnetic field strength: the $x$ profiles of the density in Fig. 2(c), which are taken for $I_{B}=5 \mathrm{~A}$ (filled squares) and $14 \mathrm{~A}$ (open circles) at $z=8 \mathrm{~cm}$ in the magnetic nozzle, show the greater density at $|x|>10 \mathrm{~cm}$ for $I_{B}=5$ A compared with that obtained for the $14 \mathrm{~A}$ case. This shows that some of the plasma within the nozzle diffuses across the field lines and is lost radially. This effect will be enhanced downstream of the source exit as the magnetic field strength weakens. In addition, as the mean-free path for ion-neutral charge exchange collisions in the nozzle is about 5 to $10 \mathrm{~cm}$ for the $0.8 \mathrm{mTorr}$ argon plasma, the cold ions resulting from this collisional process would be more magnetized and yield an increase in the plasma density (due to less radial plasma loss). This effect would contribute to the magnetic nozzle approaching an ideal nozzle when increasing the magnetic field strength.

Measurements of the plasma density upstream within the source $(z=-12 \mathrm{~cm})$ and downstream within the nozzle $(z=30 \mathrm{~cm})$ are carried out as a function of $I_{B}$, respectively, and the results are plotted in Figs. 3(a) and 4(a) as filled circles. When the magnetic field strength is increased from 0 to $14 \mathrm{~A}$, the downstream density increases
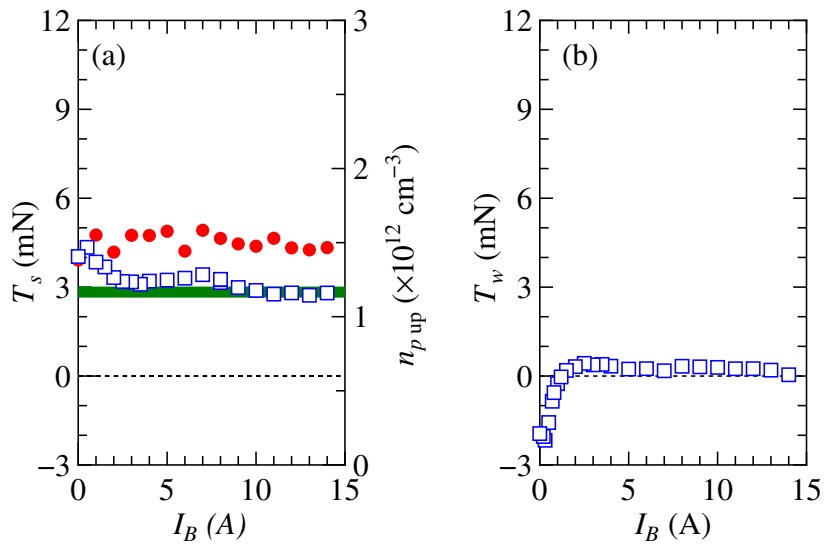

FIG. 3 (color online). (a) Measured $T_{s}$ (open squares) and the plasma density (filled circles) at $z=-12 \mathrm{~cm}$ as a function of $I_{B}$. $T_{s}$ calculated from the measured upstream electron pressure profile for $I_{B}=14 \mathrm{~A}$ is shown by the solid line. (b) Measured $T_{w}$ as a function of $I_{B}$. The dotted lines in Figs. 3(a) and 3(b) show the zero level of axial force. The typical error in the force measurement is within $\pm 5 \%$.
[Fig. 4(a)], while the upstream density is almost constant [Fig. 3(a)], demonstrating the decoupling between plasma production and plasma expansion in the magnetic nozzle. Having achieved direct and independent control of plasma expansion and cross-field diffusion solely with the solenoid current external parameter, experimental data on momentum are acquired with the aim of investigating magnetic nozzle physics and validating reported theories.

Momentum emitted from the plasma source system is equal in magnitude and opposite in direction to the force exerted on the source structure. Electron pressure forces onto the axial boundary (back plate) and magnetic nozzle have been incorporated in a previously described onedimensional model [5], where the force onto the magnetic nozzle has been derived based on area expansion of the plasma cross section [5]. The strong effect of the Lorentz force due to the radial magnetic field and the electron diamagnetic drift current has been experimentally observed and quantified [13], and the equivalence between a physical area expansion effect and the Lorentz force has been mathematically demonstrated [12]. The total force $T_{\text {total }}$ onto the bounded plasma system including the magnetic nozzle is given by $T_{\text {total }}=T_{s}+T_{w}+T_{B}$, where $T_{s}$ is the static electron pressure force exerted on the axial boundary, $T_{w}$ is the axial momentum delivered by the ions lost to the radial wall boundary, and $T_{B}$ is the Lorentz force onto the magnetic nozzle as shown by the arrows in Fig. 1(a). According to theoretical models reported in Refs. [5,12,13], these terms are presently derived assuming an axisymmetric plasma, negligible electron inertia, negligible radial ion inertia, and cold ions as

$$
T_{s}=2 \pi \int_{0}^{r_{s}} r p_{e}\left(r, z_{0}\right) d r
$$
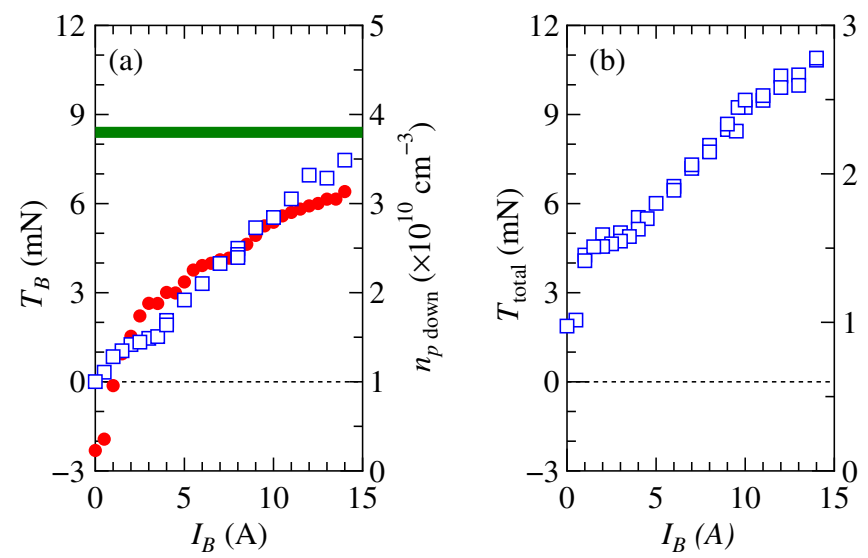

FIG. 4 (color online). (a) Measured $T_{B}$ (open squares) and the plasma density (filled circles) at $z=30 \mathrm{~cm}$ as a function of $I_{B}$. $T_{B_{\text {ideal }}}$ calculated with the ideal magnetic nozzle expansion is shown by the solid line. (b) Measured $T_{\text {total }}$ as a function of $I_{B}$. The dotted lines in Figs. 4(a) and 4(b) show the zero level of axial force. The typical error in the force measurement is within $\pm 5 \%$. 


$$
\begin{aligned}
T_{w} & =-2 \pi \int_{z_{\text {end }}}^{0} r_{s} m n_{w} u_{r}\left(r_{s}, z\right) u_{z}\left(r_{s}, z\right) d z, \\
T_{B} & =-2 \pi \int_{0}^{z} \int_{0}^{r_{p}(z)} r \frac{B_{r}(r, z)}{B_{z}(r, z)} \frac{\partial p_{e}(r, z)}{\partial r} d r d z, \\
& \simeq-\int_{0}^{z}\left\langle p_{e}(z)\right\rangle A(z) \frac{1}{B_{z 0}} \frac{\partial B_{z 0}}{\partial z} d z,
\end{aligned}
$$

where Eq. (4) is derived using the paraxial approximation $B_{z}(r, z) \simeq B_{z}(0, z) \equiv B_{z 0}$ in the one-dimensional model [12]. $r_{s}, r_{p}, p_{e}, z_{0}, n_{w}, u$ are the source radius, plasma radius, electron pressure $\left(=n_{p} k_{B} T_{e}\right.$, where $n_{p}, k_{B}$, and $T_{e}$ are the plasma density, Boltzmann constant, and electron temperature), maximum pressure position, ion density at the radial boundary, and ion velocity, respectively. $\left\langle p_{e}(z)\right\rangle$ and $A(z)$ are the radially averaged electron pressure and plasma cross section area, respectively [12].

$T_{s}$ can be calculated from the measured radial profile of the plasma density and electron temperature at $z_{0}$, which corresponds to the data in Fig. 2(b). The value of $T_{s}$ calculated for $I_{B}=14 \mathrm{~A}$ is $\sim 2.8 \mathrm{mN}$ and is indicated by a horizontal solid line in Fig. 3(a). Previous theories have neglected $T_{w}$ and assumed that the radially lost ions are axially slow ions and deliver no significant axial momentum [11], which will be discussed later based on the first direct measurement of $T_{w}$. Considering an "ideal" magnetic nozzle expansion with no radial plasma loss (no cross-field diffusion) and with plasma radius increase of the form $r_{p}(z)=r_{s} \sqrt{B_{\text {exit }} / B_{z 0}},\left\langle p_{e}(z)\right\rangle A(z)$ should be conserved along $z$ and equal to the value at the source exit $\left\langle p_{\text {exit }}\right\rangle A_{\text {exit }}$. Given the radial profile of electron pressure measured at the source exit $(z=0 \mathrm{~cm})$, the theoretical limit of the electron-diamagnetic-induced force $T_{B}$ onto the magnetic nozzle can be obtained from Eq. (4). Using the magnetic field on axis shown in Fig. 1(b) and the radial profiles of plasma density and electron temperature measured for $I_{B}=14 \mathrm{~A}$ at the source exit (not shown here), $r_{p}$ and $T_{B}$ are calculated from the ideal nozzle model and shown by dashed and solid lines in Fig. 1(c), respectively. The theoretical limit of $T_{B}$ should be that computed for a plasma radius approaching the chamber radius of $30 \mathrm{~cm}$, which corresponds to $z=29.3 \mathrm{~cm}$. The value of $T_{B_{\text {ideal }}}$ obtained at $z=29.3 \mathrm{~cm}$ in Fig. 1(c) is indicated by a solid horizontal line in Fig. 4(a) and is about $8.4 \mathrm{mN}$.

To verify $T_{s}$ and $T_{w}$ described in the model [Eq. (1) and (2)] and the approach to the theoretical limit of $T_{B}$ [Eq. (4)], direct measurements of the axial forces are performed as a function of $I_{B}$ using the pendulum force balance. As the force components are exerted on the axial $\left(T_{s}\right)$ and radial $\left(T_{w}\right)$ boundaries and the magnetic field lines produced by the solenoid $\left(T_{B}\right)$, these can be solely measured by connecting either the source back plate or glass source tube or solenoid to the force balance, respectively. The total force $T_{\text {total }}$ is also measured by connecting all three source components to the balance (the rf antenna is not in contact with the glass tube and not connected to the balance). The pendulum is moved to the negative $z$ direction for positive force corresponding to the direction of the arrows $\left(T_{s}, T_{w}, T_{B}\right)$ in Fig. 1(a), and vice versa. The displacement is measured using the laser sensor $(0.1-\mu \mathrm{m}$ resolution and $312.5-\mathrm{Hz}$ sampling rate), and the force is obtained using the calibration coefficient $(0.621,0.057$, 0.087 , and $0.506 \mathrm{mN} / \mu \mathrm{m}$ for the measurements of $T_{\text {total }}$, $T_{s}, T_{w}$, and $T_{B}$, respectively). The typical error in the force measurement is within $\pm 5 \%$.

The measured $T_{s}$ and $T_{w}$ are plotted by open squares in Figs. 3(a) and 3(b), respectively. $T_{s}$ versus $I_{B}$ (open squares) is found to be fairly constant since the maximum electron pressure is invariant with $I_{B}$ as shown by the filled circles in Fig. 3(a). The value of $T_{s}$ is in good agreement with the value $T_{s}$ calculated from the measured electron pressure for $I_{B}=14 \mathrm{~A}$, using Eq. (1) and shown by the solid line. $T_{w}$ is close to zero above $2 \mathrm{~A}$ solenoid current in Fig. 3(b), while a negative value of $T_{w}$ is detected with no or weak magnetic field less than about 1 A. Figure 2(a) shows that with no magnetic field the axial density profile is asymmetric with a strong density decay (hence a plasma potential decay) on axis from the antenna position $(z \sim$ $-12 \mathrm{~cm}$ ) to the source exit. This axial profile is an indication that more ions are lost into the radial boundary and/ or axially accelerated over the downstream half region of the source $(-12<z<0 \mathrm{~cm})$ compared with the upstream region $(-20<z<-12 \mathrm{~cm})$. The large density gradient near the open end of the source for no magnetic field suggests a larger axial electric field resulting in a net negative force delivered by the radially lost ions, since the measured electron temperature is uniform on axis within the source cavity. For a magnetic field above $2 \mathrm{~A}$ current, a symmetric axial density profile is measured and a zero value for $T_{w}$ is obtained, confirming that neglecting $T_{w}$ in the magnetic nozzle plasma is clearly validated.

The measured $T_{B}$ and $T_{\text {total }}$ are plotted by open squares in Figs. 4(a) and 4(b), respectively. Figure 4(a) clearly shows the increase in $T_{B}$ with increasing $I_{B}$, i.e., the magnetic field strength. The increase in the magnetic field strength by the solenoid current does not change the $B_{r} / B_{z}$ factor in Eq. (3) but yields an increase in plasma density (or electron pressure) in the magnetic nozzle as seen in the measurements of Fig. 2(a) and of the filled circles in Fig. 4(a), which originates from inhibition of the cross-field diffusion. Hence the absolute value of $\partial p_{e} / \partial r$ in Eq. (3) and of $T_{B}$ is increased. The experimentally measured $T_{B}$ is found to approach the theoretical upper limit $T_{B_{\text {ideal }}}$ [bold solid line in Fig. 4(a)] derived from the ideal nozzle approximation. Since $T_{B}$ is experimentally decoupled from $T_{s}$ (a constant invariant with $I_{B}$ ) and $T_{w}$ (neglected in the presence of an applied magnetic field), an increase of $T_{B}$ will result in an increase of $T_{\text {total }}=T_{s}+T_{w}+T_{B}$. This is experimentally confirmed with the direct measurement of $T_{\text {total }}$ shown on Fig. 4(b). 
The solenoid current in the present setup is limited below $\sim 14$ A because of thermal issues. Higher currents would validate whether the system has reached the fundamental limit of an ideal nozzle but would necessitate a bigger and heavier solenoid not compatible with the presently used thrust balance.

In summary, direct measurements of axial plasma momentum components imparted from boundaries of a cylindrical plasma source and from a magnetic nozzle decoupled from plasma production are performed. It is experimentally demonstrated that the axial momentum generated by the magnetic nozzle $\left(T_{B}\right)$ increases with increasing magnetic field strength due to inhibition of the cross-field plasma diffusion and approaches the theoretical limit of an "ideal" magnetic nozzle model. The independent measurements of axial momentum exerted onto the source back plate $\left(T_{s}\right)$ and onto the radial source wall $\left(T_{w}\right)$ validate the model, which considers the maximum electron pressure onto the axial boundary and assumes a zero net axial momentum on the the radial boundary.

The authors would like to thank Professor A. Fruchtman of the Holon Institute of Technology for many useful discussions. A part of this work is financially supported by JSPS (Young Scientist A 22684031 and Scientific Research B 25287150), the Sumitomo Foundation, and the JST A-step program. *kazunori@ecei.tohoku.ac.jp

Permanent address: Department of Electrical Engineering, Tohoku University, Sendai 980-8579, Japan.

[1] K. Stasiewicz, Phys. Rev. Lett. 96, 175003 (2006).

[2] A. Ciardi, S. V. Lebedev, A. Frank, F. Suzuki-Vidal, G. N. Hall, S. N. Bland, A. Harvey-Thompson, E. G. Blackman, and M. Camenzind, Astrophys. J. 691, L147 (2009).

[3] Y. Wang, F. S. Wei, X. S. Feng, S. H. Zhang, P. B. Zuo, and T. R. Sun, Phys. Rev. Lett. 105, 195007 (2010).

[4] M. Koepke, Rev. Geophys. 46, RG3001 (2008).

[5] A. Fruchtman, Phys. Rev. Lett. 96, 065002 (2006).

[6] A. V. Arefiev and B. N. Breizman, Phys. Plasmas 12, 043504 (2005).

[7] E. Ahedo and M. Merino, Phys. Plasmas 17, 073501 (2010).

[8] E. Ahedo and M. Merino, Phys. Plasmas 18, 053504 (2011).

[9] A. Fruchtman, Plasma Sources Sci. Technol. 18, 025033 (2009).

[10] S. Rao and N. Singh, Phys. Plasmas 19, 093507 (2012).

[11] A. Fruchtman, IEEE Trans. Plasma Sci. 36, 403 (2008).

[12] A. Fruchtman, K. Takahashi, C. Charles, and R. W. Boswell, Phys. Plasmas 19, 033507 (2012).

[13] K. Takahashi, T. Lafleur, C. Charles, P. Alexander, and R. W. Boswell, Phys. Rev. Lett. 107, 235001 (2011).

[14] K. Takahashi, Rev. Sci. Instrum. 83, 083508 (2012).

[15] T.E. Sheridan, Phys. Plasmas 7, 3084 (2000). 